\title{
Three Multiple-Pulse Operation States of an All-Normal-Dispersion Dissipative Soliton Fiber Laser
}

\author{
Liqiang Zhang, ${ }^{1}$ Zhiyong Pan, ${ }^{2}$ Zhuang Zhuo, ${ }^{1}$ and Yunzheng Wang ${ }^{1}$ \\ ${ }^{1}$ School of Information Science and Engineering, Shandong University, 27 Shanda Nanlu, Jinan 250100, China \\ ${ }^{2}$ No. 23 Research Institute of China Electronic Technology Corporation Group, 230 Tieshan Road, Shanghai 201900, China \\ Correspondence should be addressed to Zhuang Zhuo; zhuozhuang@sdu.edu.cn
}

Received 27 December 2013; Accepted 14 February 2014; Published 17 March 2014

Academic Editor: Yu S. Kivshar

Copyright (c) 2014 Liqiang Zhang et al. This is an open access article distributed under the Creative Commons Attribution License, which permits unrestricted use, distribution, and reproduction in any medium, provided the original work is properly cited.

\begin{abstract}
Multiple-pulse operation states of an all-normal-dispersion Yb-doped double-clad dissipative soliton fiber laser are investigated in this paper. The proposed laser can deliver harmonic mode-locked pulses, bound states of dissipative solitons, and dual-wavelength dual-pulses. Stable second-harmonic and third-harmonic mode-locked pulse trains are obtained with the output power of $1.39 \mathrm{~W}$ and $1.46 \mathrm{~W}$, respectively, and the corresponding single pulse energies are $12.1 \mathrm{~nJ}$ and $8.5 \mathrm{~nJ}$. With the adjustment of pump power and the wave plates, the fiber laser generates bound states of two or three dissipative solitons. Moreover, a dual-wavelength dual-pulse state is presented, where the output pulses from the nonlinear polarization rotation rejection port consists of the leading and trailing edges of the pulses circulating in the cavity.
\end{abstract}

\section{Introduction}

Passively mode-locked fiber lasers as potential sources of the ultrashort optical pulses have been intensively investigated [1-3]. Depending on the cavity design, the net dispersion of the fiber laser can be normal or anomalous. Fiber lasers with net anomalous group velocity dispersion deliver conventional solitons with little chirp. The formation of the conventional solitons results from the balance between nonlinearity and dispersion. Dissipative solitons generate in net- or all-normal dispersion fiber lasers and have attracted considerable attention recently as they are able to deliver high energy pulses $[4,5]$. The formation of dissipative solitons is a result of mutual interactions among dispersion, nonlinearity, laser gain, and loss, where the balance between gain and loss plays a dominant role [6].

Depending on the experimental condition, fiber lasers can operate in different regimes. Generally it is observed in multiple pulsing regimes, where the pulses scatter randomly in the cavity. Under certain conditions, the intervals between multiple pulses can be equalized. Such an operation state is known as harmonic mode-locking, which was firstly reported by Richardson et al. in 1991 [7]. An immediate advantage of the harmonically mode-locked laser is that their repetition rate could be many times the cavity fundamental repetition rate; thus high repetition rate pulses could be obtained [8]. Another multiple-pulses operation state is bound state or soliton molecules, where multiple pulses stay very close to each other. Bound states of solitons was predicted in the coupled nonlinear Schödinger equations [9] and the quintic complex Ginzburg-Landau equation [10, 11], and the formation of bound solitons was explained as a result of direct soliton interaction [12]. Both harmonic mode-locking [13-17] and bound states [18-23] have been intensively investigated in the last two decades. Harmonic mode-locking and bound states of conventional solitons [13, 18], stretched pulses [19], parabolic pulses [21], and dissipative solitons [22] have all been reported. Stable harmonic mode-locking of dissipative solitons in all-normal-dispersion fiber lasers was firstly reported in 2010 [24]. Recently, Liu et al. [25] investigated the formation and evolution of bound dissipative pulses in the all-normal dispersion Yb-fiber laser. However, as most of the fiber lasers mentioned above were pumped in-core by single mode lasers, the output power and pulse energy were very low. In 2009, Kieu et al. [26] reported an allnormal-dispersion dissipative soliton fiber laser employing 


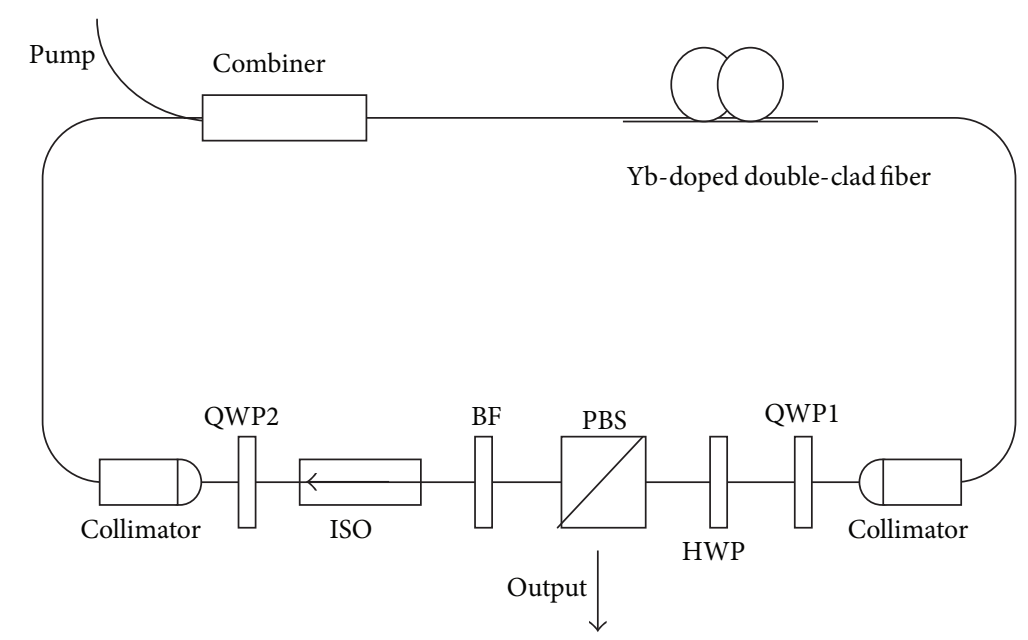

FIGURE 1: Experimental setup of the Yb-doped double-clad dissipative soliton fiber laser.

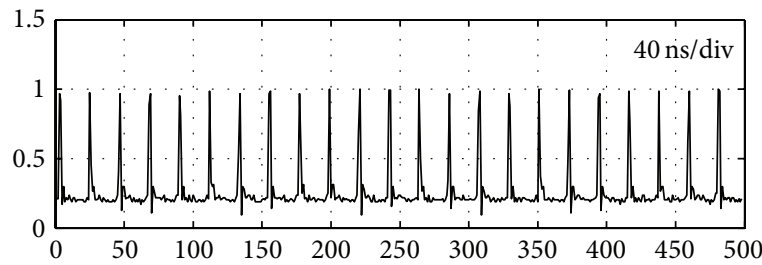

(a)

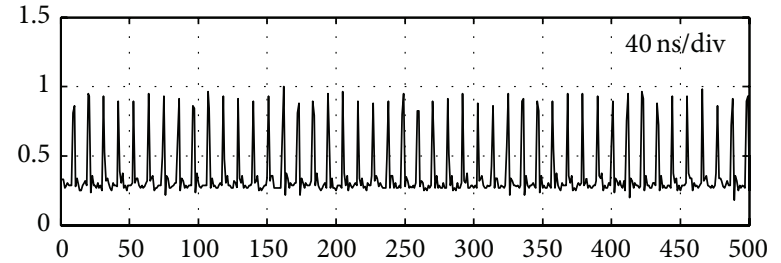

(b)

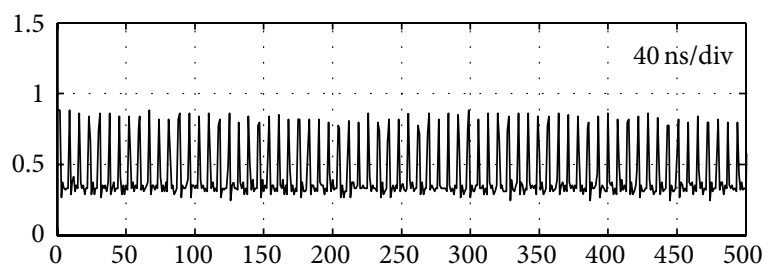

(c)

FIGURE 2: Measured mode-locked pulse trains at different repetition rates. (a) Fundamental mode-locking at 57.5 MHz. (b) Second-harmonic mode-locking at $115 \mathrm{MHz}$. (c) Third-harmonic mode-locking at $172.5 \mathrm{MHz}$.

double-clad gain fiber with the average output power of $2.2 \mathrm{~W}$, corresponding to pulse energy of $31 \mathrm{~nJ}$. It is of particular interest to know whether harmonic mode-locking and bound states of dissipative solitons can be obtained in these wattlevel all-normal-dispersion fiber lasers.

In addition to harmonic mode-locking and bound states, there is another multiple-pulse operation state in dissipative soliton fiber lasers. In fiber lasers mode-locked by the nonlinear polarization rotation, the output from the polarization beam splitter (PBS) rejection port plays the role of nonlinear absorption of the artificial saturable absorber. The rejected pulse shape varied with the wave plates settings [27]. Under certain conditions, the central region of the pulse passes through the PBS losslessly, and the rejected pulse consists of the leading and trailing edges of the intracavity pulse, which can be regarded as two subpulses. In [28], we gave a detailed report on this phenomenon. Further investigation on this phenomenon has been made and confirmed it is a dualwavelength dual-pulse operation state.

In this paper, we demonstrate three multiple-pulse operation states mentioned above from an all-normal-dispersion Yb-doped double-clad dissipative soliton fiber laser. Depending on the pump power and the orientations of wave plates, laser can operate in diverse states: harmonic mode-locking, bound states, and so called dual-wavelength dual-pulse operation state.

\section{Experimental Setup}

Figure 1 shows the setup of the all-normal-dispersion $\mathrm{Yb}$ doped double-clad dissipative soliton fiber laser demonstrated in this paper. A section of 2-m Yb-doped doubleclad fiber with a core diameter of $10 \mu \mathrm{m}$ was used as the gain medium. Cladding absorption at $976 \mathrm{~nm}$ was about $6.5 \mathrm{~dB} / \mathrm{m}$. 


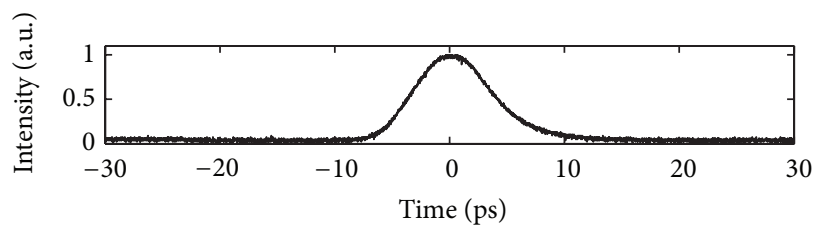

(a)

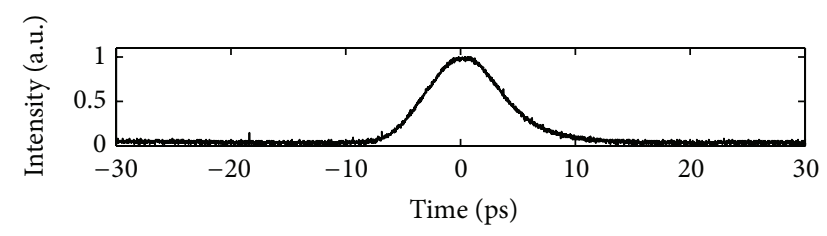

(b)

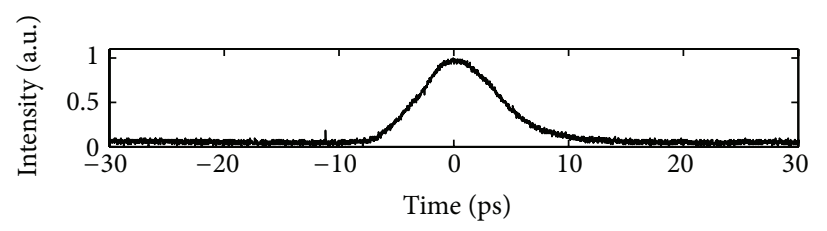

(c)

FIGURE 3: Autocorrelation traces of the (a) fundamental mode-locked, (b) second-harmonic mode-locked, and (c) third-harmonic modelocked pulses.

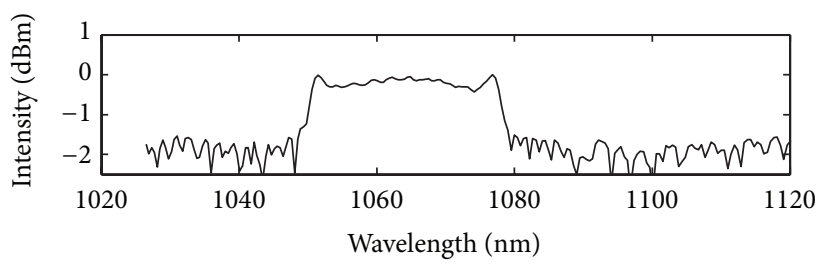

(a)

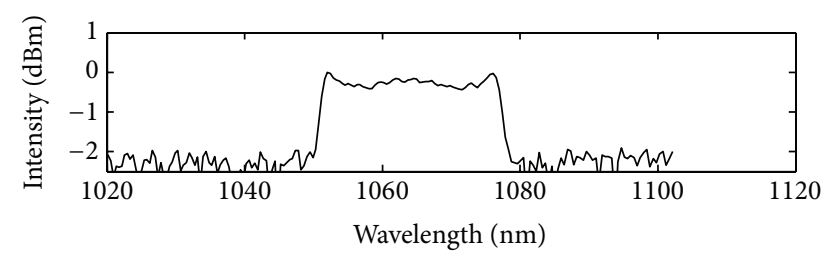

(b)

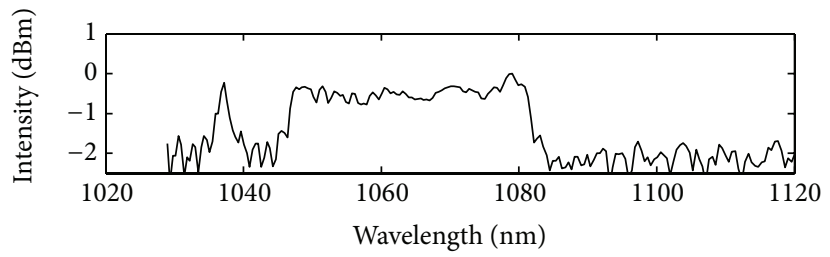

(c)

FIGURE 4: Optical spectra of the (a) fundamental mode-locked, (b) second-harmonic mode-locked, and (c) third-harmonic mode-locked pulses.

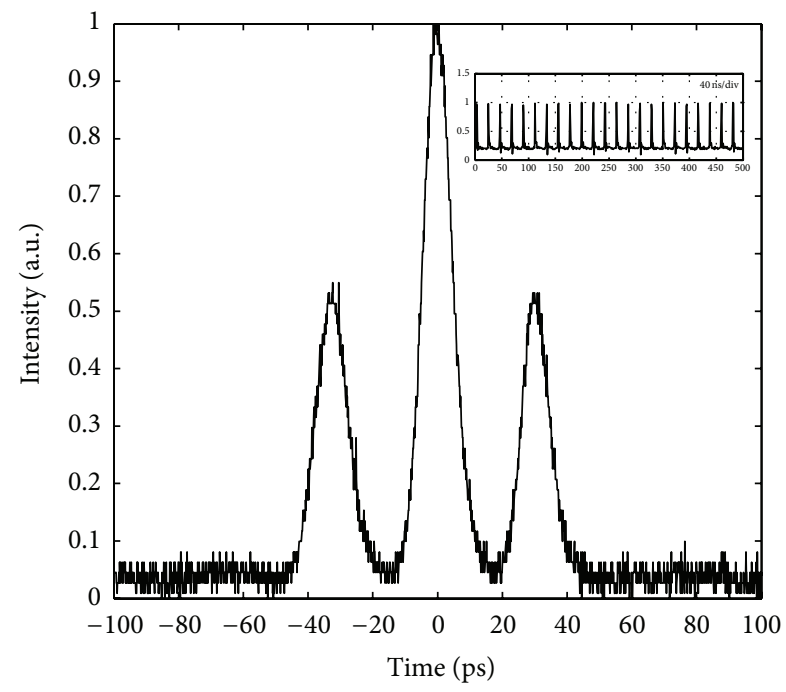

(a)

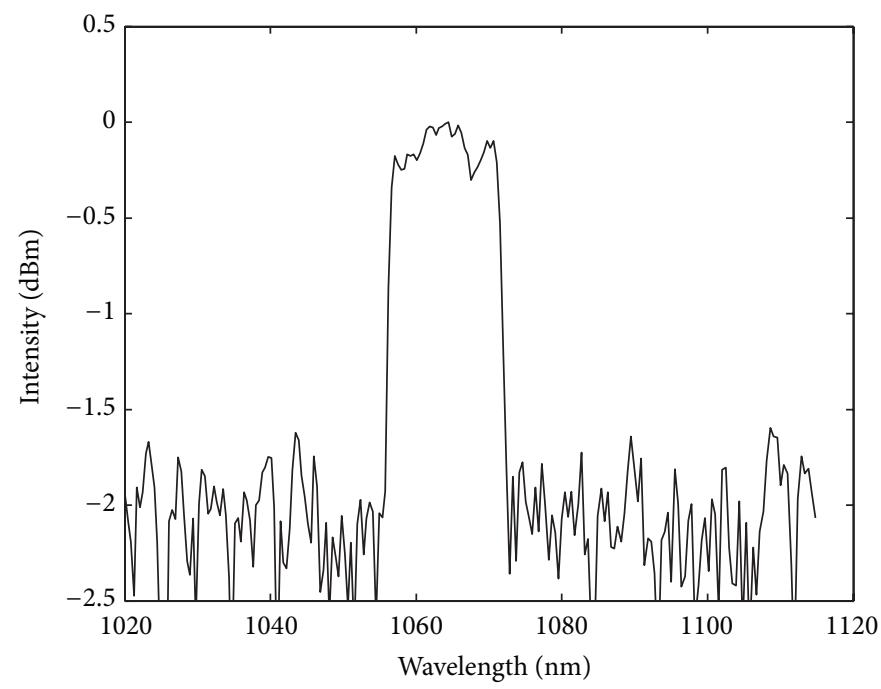

(b)

FIGURE 5: Characteristics of a bound state of dissipative solitons. (a) Autocorrelation trace. Inset, pulse train. (b) Optical spectrum. 


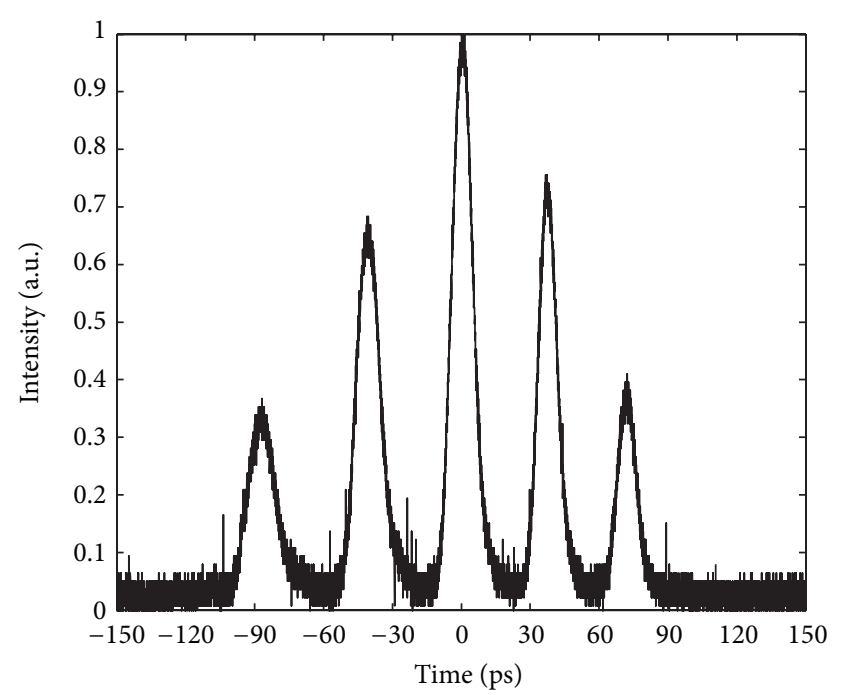

FIGURE 6: Autocorrelation trace of the bound state of three dissipative solitons.

A fiber coupled diode laser with central wavelength of $975 \mathrm{~nm}$ was used as the pump laser. The pump light was coupled into the gain fiber through a pump/signal combiner. The fiber laser was mode-locked by nonlinear polarization rotation. Two quarter wave plates (QWP1, QWP2), one half wave plate (HWP), and a polarization beam splitter (PBS) were used in the resonator. A birefringent quartz plate of $4.09 \mathrm{~mm}$ thickness, inserted between the PBS and the isolator (ISO), provided a sinusoidal spectral transmission. The bandwidth around $1060 \mathrm{~nm}$ of the filter was about $15 \mathrm{~nm}$. All the fibers have normal group velocity dispersion around $1.06 \mu \mathrm{m}$. The total length of the cavity was about $3.6 \mathrm{~m}$, and the overall dispersion for the whole cavity was about $0.15 \mathrm{ps}^{2}$. The output was taken directly from the PBS rejection port, which was analyzed with an optical spectrum analyzer (Q8344A) and a commercial optical autocorrelator (FR-103 XL). A $1 \mathrm{GHz}$ digital oscilloscope (TDS 7104) together with a $1.5 \mathrm{GHz}$ photo-detector was used to monitor the mode-locked pulse trains.

\section{Experimental Results and Discussion}

3.1. Harmonic Mode-Locking. The threshold pump power for mode locking was $2.24 \mathrm{~W}$. With the adjustment of the pump power and the wave plates, stable fundamental mode-locked, second-harmonic mode-locked, and third-harmonic modelocked pulse trains were obtained. Figures 2, 3, and 4 are the pulse trains, autocorrelation traces, and optical spectra at different repetition rates. The corresponding pump power is $3.11 \mathrm{~W}, 4.28 \mathrm{~W}$, and $6.17 \mathrm{~W}$, respectively. The fundamental repetition rate is $57.5 \mathrm{MHz}$, which matches well with the cavity round-trip time. As shown in Figure 3(a), the fundamental mode-locked pulse duration is estimated to be $5.6 \mathrm{ps}$ if a Gaussian-shape pulse is assumed. The pulse durations change slightly with the repetition rate. The steep sides at the edges

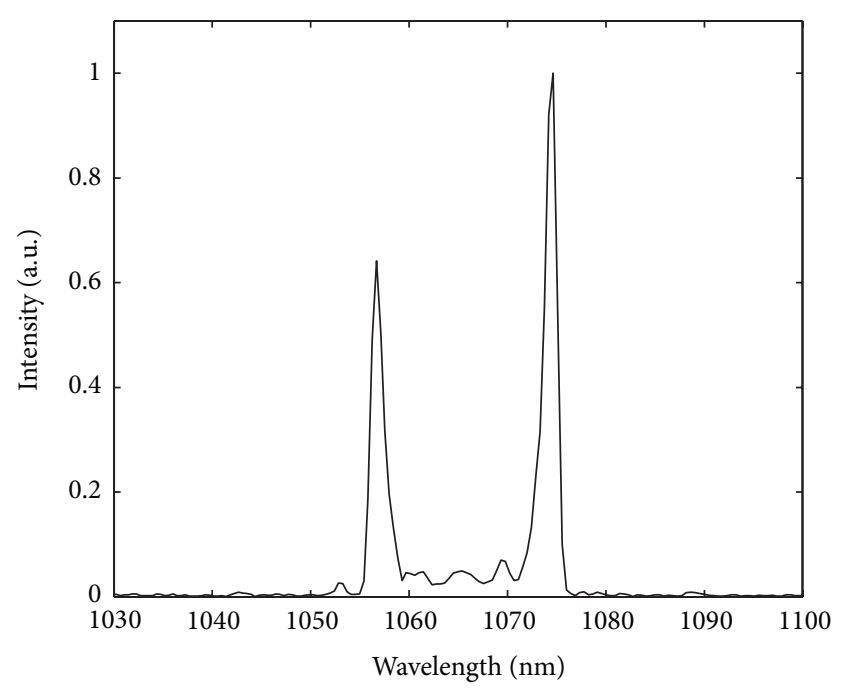

(a)

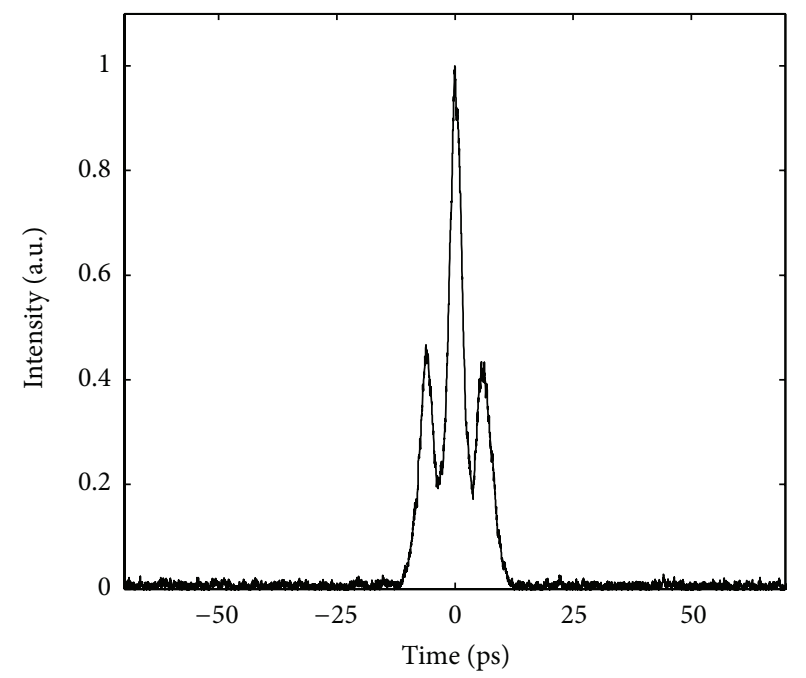

(b)

Figure 7: Characteristics of the mode-locked pulses operating in dual-wavelength dual-pulse state. (a) Optical spectrum. (b) Autocorrelation trace.

of the spectra (Figure 4) are considered as a typical characteristic of the dissipative solitons. The central wavelength of fundamental mode-locked pulse is $1064.2 \mathrm{~nm}$, and the edgeto-edge bandwidth is $26.9 \mathrm{~nm}$. The peak around $1037.2 \mathrm{~nm}$ in Figure 4(c) means the optical spectrum of the third-order mode-locked pulse contains a cw spectral component.

The measured output power was $1.07 \mathrm{~W}, 1.39 \mathrm{~W}$, and $1.46 \mathrm{~W}$, with single pulse energy of $18.6 \mathrm{~nJ}, 12.1 \mathrm{~nJ}$, and $8.5 \mathrm{~nJ}$, respectively. The obtained average power and pulse energy were about 31 times and 10 times the results obtained in an all-normal-dispersion Yb-doped fiber laser with the same configuration [17]. The higher average power and pulse energy resulted from the use of the double-clad gain fiber.

3.2. Bound States of Dissipative Solitons. A bound state of dissipative solitons was observed at the pump power of 


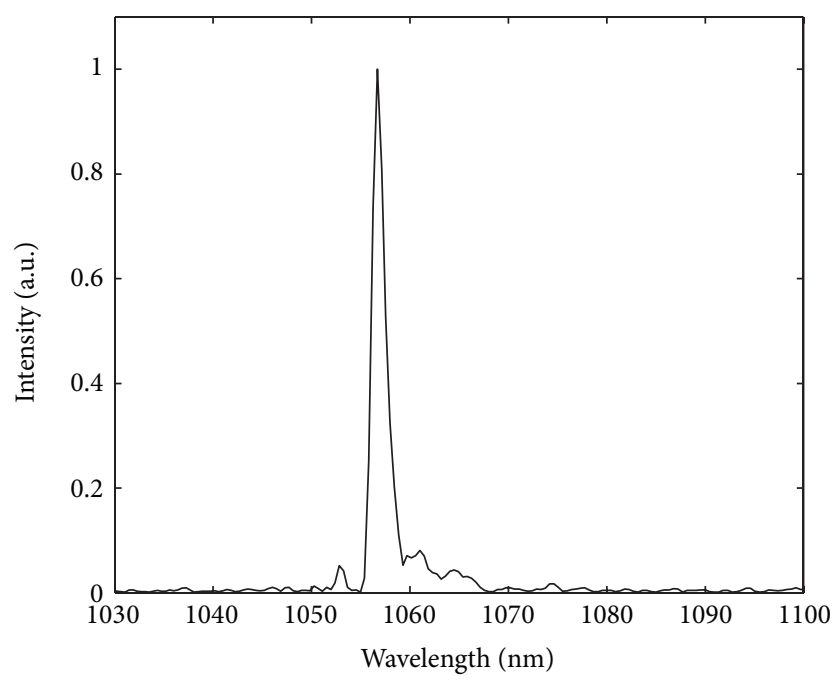

(a)

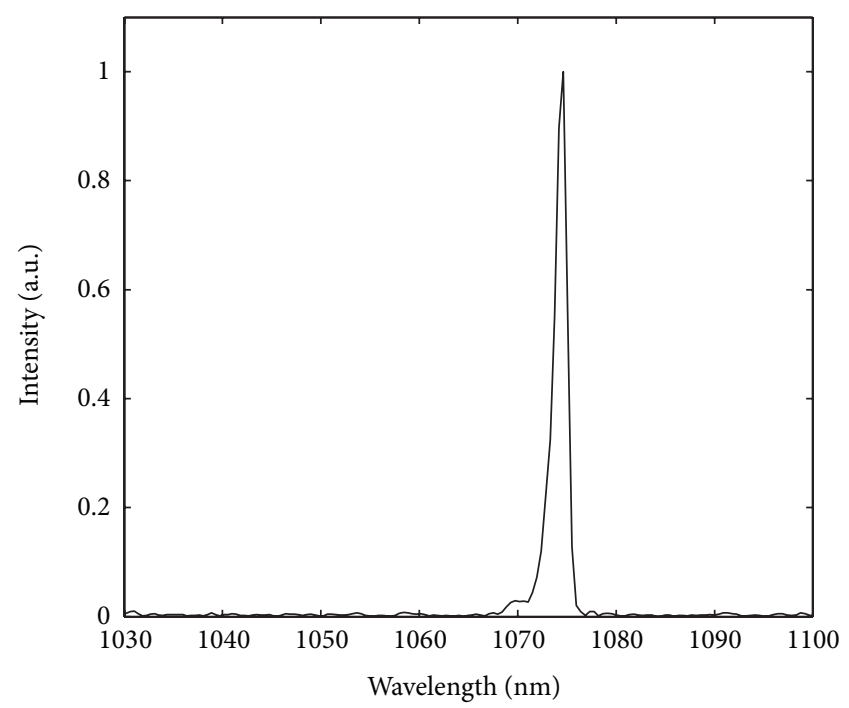

(c)

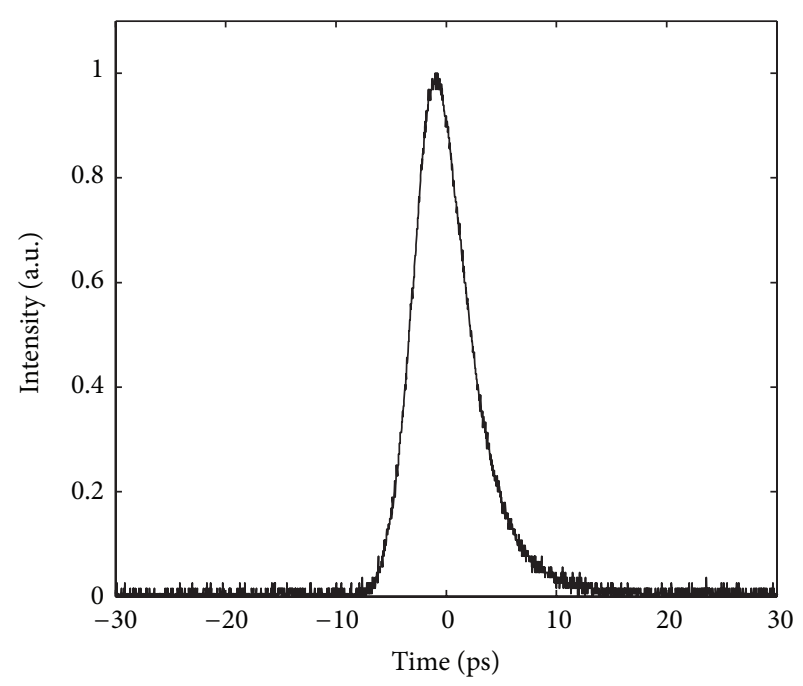

(b)

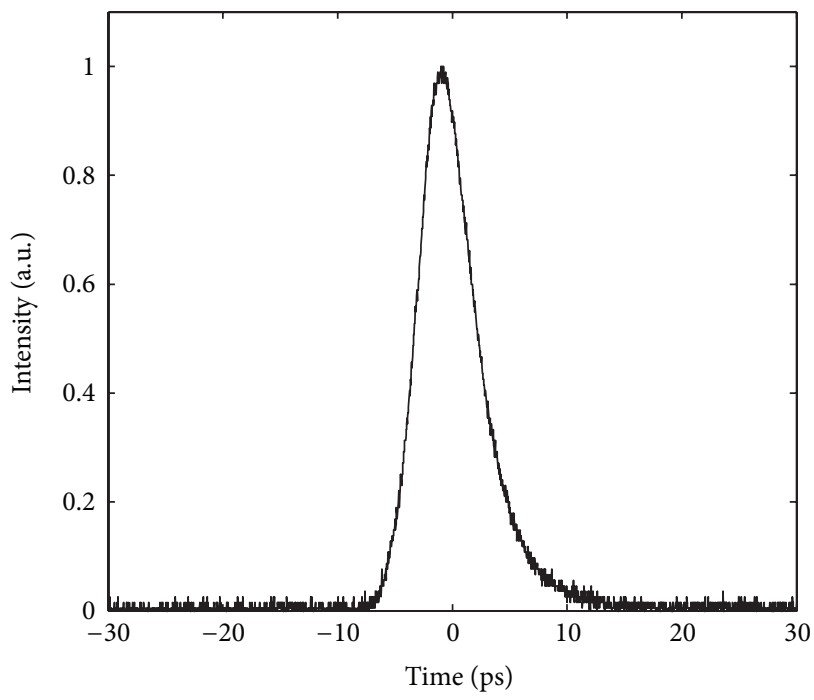

(d)

FIGURE 8: Characteristics of the output pulses after filtering. (a) and (b) Optical spectrum and autocorrelation trace of the pulses with the light around $1074 \mathrm{~nm}$ are filtered out. (c) and (d) Optical spectrum and autocorrelation trace of the pulses with the light around $1056.8 \mathrm{~nm}$ are filtered out.

$2.59 \mathrm{~W}$, and the results are shown in Figure 5. Figure 5(a) gives the measured autocorrelation trace of the pulses. The pulse duration is about $7.4 \mathrm{ps}$ under a Gaussian profile assumption. The pulse separation between the bound solitons is measured to be about $32 \mathrm{ps}$, which is roughly 4.3 times of the pulse duration. The height ration of the three peaks is $1: 2: 1$, indicating that the two bound dissipative solitons are identical. The inset in Figure 5(a) is the oscilloscope trace of the pulse train. The repetition rate is $57.5 \mathrm{MHz}$, which shows that only one group of bound dissipative solitons exists in the cavity. The edge-to-edge width of the optical spectrum shown in Figure 5(b) is $14.47 \mathrm{~nm}$, narrower than the results of harmonic mode-locking state. Different from the spectra of bound gain-guided solitons [20] and dissipative-soliton molecules [22], regular modulation on the whole spectral profile is not obvious, which suggests that the relative phase between the pulses is rotating [29].
It is thus natural to expect that multiple bound states would be obtained. Indeed, we observed the emission of a bound state of three dissipative solitons at the pump power of $4.28 \mathrm{~W}$. The measured autocorrelation trace is shown in Figure 6 . There are five peaks, with an intensity ratio of about $1: 2: 3: 2: 1$. The pulse duration is $7.8 \mathrm{ps}$ and the pulse separation between the neighboring solitons is $28.3 \mathrm{ps}$. The peak-to-peak separation is about 3.6 times that of the pulse duration. The optical spectrum of the bound state of the three dissipative solitons is similar to that in Figure 5(b). The average output power is $1.66 \mathrm{~W}$, corresponding to single pulse energy of $9.6 \mathrm{~nJ}$.

3.3. Dual-Wavelength Dual-Pulse Operation State. In [28], we gave a systematic report on pulse splitting behavior in a dissipative soliton fiber laser with the PBS rejection port as 
the output. It was pointed that the rejected pulse would split into two subpulses under certain conditions. Here we present a further investigation on this phenomenon and demonstrate that it is a dual-wavelength operation state.

Figure 7 gives the characteristics of the mode-locked pulses. Figure 7(a) is the measured optical spectrum. The central wavelengths of the two peaks are $1056.8 \mathrm{~nm}$ and $1074.4 \mathrm{~nm}$, with the bandwidths of $1.56 \mathrm{~nm}$ and $1.5 \mathrm{~nm}$, respectively. Figure 7(b) shows the corresponding autocorrelation trace, with a peak separation of 9 ps. Partial overlapping of the three peaks in the autocorrelation trace is just because the two pulses are too close to each other.

Figure 8 gives the results when the output pulse is filtered with interference filters. The central wavelengths of the filters are $1060 \mathrm{~nm}$ and $1075 \mathrm{~nm}$, respectively. The bandwidths of both filters are $10 \mathrm{~nm}$. Figures $8(\mathrm{a})$ and $8(\mathrm{~b})$ are the optical spectrum and autocorrelation trace of the pulse when the light around $1074 \mathrm{~nm}$ was filtered out. As shown in Figure 8 (b), there remains only one pulse, with duration of 4.1 ps. Figures $8(\mathrm{c})$ and $8(\mathrm{~d})$ are the results when the light around $1056.8 \mathrm{~nm}$ was filtered out. The single pulse duration is $3.8 \mathrm{ps}$.

The formation of the dual-wavelength pulse is an intrinsic property of nonlinear polarization rotation mechanism. The light after PBS is linearly polarized. The QWP2 transfers the linearly polarized light into elliptically polarized light. In the fiber section, different parts of the pulse get different polarization evolution. The following QWP1 and HWP further change the polarization states so that different parts of the pulse experience different losses after passing through the PBS. When the nonlinear polarization rotation effect is strong enough, the central region of the pulse passes through the PBS losslessly, and the rejected pulse consists almost of all the pulse wings. As the pulse circulating in the cavity is highly chirped, the leading and trailing edges of the pulse contain different frequency components. Thus the optical spectrum spilt into two separated parts. It is worthy of noting that when we deliberately changed the cavity property of our laser, such as the total cavity length or the central operating wavelength by rotating the quartz filter, the dual-wavelength dual-pulse state could always be obtained. It thus can be thought as a generic feature of the passively mode-locked fiber lasers.

\section{Conclusion}

In conclusion, we have successfully demonstrated three multiple-pulse operation states in an all-normal-dispersion dissipative soliton fiber laser mode-locked by nonlinear polarization rotation in this paper. Through adjustment of the pump power and orientations of the wave plates, harmonic mode-locking, bound states, and dual-wavelength dual-pulse states were obtained. This work gives a more clear understanding on the characteristics of all-normaldispersion dissipative soliton fiber lasers.

\section{Conflict of Interests}

The authors declare that there is no conflict of interests regarding the publication of this paper.

\section{Acknowledgments}

The authors acknowledge the funding support from Interdisciplinary Incubation Project Foundation of Shandong University (2011JC025) and Natural Science Foundation of Shandong Province (ZR2010FM029).

\section{References}

[1] V. J. Matsas, T. P. Newson, D. J. Richardson, and D. N. Payne, "Selfstarting passively mode-locked fibre ring soliton laser exploiting nonlinear polarisation rotation," Electronics Letters, vol. 28, no. 15, pp. 1391-1393, 1992.

[2] M. Hofer, M. H. Ober, F. Haberl, and M. E. Fermann, "Characterization of ultrashort pulse formation in passively modelocked fiber lasers," IEEE Journal of Quantum Electronics, vol. 28, no. 3, pp. 720-728, 1992.

[3] K. Tamura, E. P. Ippen, H. A. Haus, and L. E. Nelson, "77-fs pulse generation from a stretched-pulse mode-locked all-fiber ring laser," Optics Letters, vol. 18, no. 13, pp. 1080-1082, 1993.

[4] A. Chong, J. Buckley, W. Renninger, and F. Wise, "All-normaldispersion femtosecond fiber laser," Optics Express, vol. 14, no. 21, pp. 10095-10100, 2006.

[5] Z. W. Xu and Z. X. Zhang, "All-normal-dispersion multiwavelength dissipative soliton Yb-doped fiber laser," Laser Physics Letters, vol. 10, no. 8, Article ID 085105, 2013.

[6] P. Grelu and N. Akhmediev, "Dissipative solitons for modelocked lasers," Nature Photonics, vol. 6, no. 2, pp. 84-92, 2012.

[7] D. J. Richardson, R. I. Laming, D. N. Payne, M. W. Phillips, and V. J. Matsas, " $320 \mathrm{fs}$ Soliton generation with passively modelocked erbium fibre laser," Electronics Letters, vol. 27, no. 9, pp. 730-732, 1991.

[8] L. M. Zhao, D. Y. Tang, T. H. Cheng et al., "Passive harmonic mode locking of soliton bunches in a fiber ring laser," Optical and Quantum Electronics, vol. 40, no. 13, pp. 1053-1064, 2008.

[9] B. A. Malomed, "Bound solitons in the nonlinear SchrödingerGinzburg-Landau equation," Physical Review A, vol. 44, no. 10, pp. 6954-6957, 1991.

[10] B. A. Malomed, "Bound solitons in coupled nonlinear Schrödinger equations," Physical Review A, vol. 45, no. 12, pp. R8321-R8323, 1992.

[11] N. N. Akhmediev, A. Ankiewicz, and J. M. Soto-Crespo, "Multisoliton solutions of the complex ginzburg-landau equation," Physical Review Letters, vol. 79, no. 21, pp. 4047-4051, 1997.

[12] D. Y. Tang, B. Zhao, D. Y. Shen, C. Lu, W. S. Man, and H. Y. Tam, "Bound-soliton fiber laser," Physical Review A, vol. 66, no. 3, Article ID 033806, 2002.

[13] Y. C. Meng, S. M. Zhang, X. L. Li, H. F. Li, J. Du, and Y. P. Hao, "Passive harmonically mode-locked fiber laser with low pumping power based on a graphene saturable absorber," Laser Physics Letters, vol. 9, no. 7, pp. 537-541, 2012.

[14] S. Zhou, D. G. Ouzounov, and F. W. Wise, "Passive harmonic mode-locking of a soliton $\mathrm{Yb}$ fiber laser at repetition rates to 1.5 GHz," Optics Letters, vol. 31, no. 8, pp. 1041-1043, 2006.

[15] J. Du, S. M. Zhang, H. F. Li, Y. C. Meng, X. L. Li, and Y. P. Hao, "L-band passively harmonic mode-locked fiber laser based on 
a graphene saturable absorber," Laser Physics Letters, vol. 9, no. 12, pp. 896-900, 2012.

[16] C. Lecaplain and P. Grelu, "Multi-gigahertz repetition-rateselectable passive harmonic mode locking of a fiber laser," Optics Express, vol. 21, no. 9, pp. 10897-10902, 2013.

[17] L. Kong, X. Xiao, and C. Yang, "Passive harmonic mode locked all-normal-dispersion Yb-doped fibre lasers," Chinese Physics B, vol. 20, no. 2, Article ID 024207, 2011.

[18] D. Y. Tang, W. S. Man, H. Y. Tam, and P. D. Drummond, "Observation of bound states of solitons in a passively modelocked fiber laser," Physical Review A, vol. 64, no. 3, Article ID 033814, 2001.

[19] B. Ortaç, A. Hideur, and M. Brunel, "Binding widely-separated pulses with a passively mode-locked Yb-doped double-clad fiber laser," Applied Physics B, vol. 79, no. 2, pp. 185-192, 2004.

[20] L. M. Zhao, D. Y. Tang, X. Wu, D. J. Lei, and S. C. Wen, "Bound states of gain-guided solitons in a passively mode-locked fiber laser," Optics Letters, vol. 32, no. 21, pp. 3191-3193, 2007.

[21] B. Ortaç, A. Hideur, M. Brunel et al., "Generation of parabolic bound pulses from a Yb-fiber laser," Optics Express, vol. 14, no. 13, pp. 6075-6083, 2006.

[22] X. Liu, "Dynamic evolution of temporal dissipative-soliton molecules in large normal path-averaged dispersion fiber lasers," Physical Review A, vol. 82, no. 6, Article ID 063834, 2010.

[23] J. S. Peng, L. Zhan, S. Luo, and Q. Shen, "Passive harmonic mode-locking of dissipative solitons in a normal-dispersion Erdoped fiber laser," Journal of Lightwave Technology, vol. 31, no. 16, pp. 3009-3014, 2013.

[24] D. Liu, X. Zhu, C. Wang et al., "Passive harmonically modelocked Yb3+-doped fiber laser free from anomalous dispersion," IEEE Photonics Technology Letters, vol. 22, no. 23, pp. 1726-1728, 2010.

[25] X. Liu, H. Wang, Y. Wang et al., "Bound dissipative-pulse evolution in the all-normal dispersion fiber laser using a $45^{\circ}$ tilted fiber grating," Laser Physics Letters, vol. 10, Article ID 095103, 2013.

[26] K. Kieu, W. H. Renninger, A. Chong, and F. W. Wise, "Sub-100 fs pulses at watt-level powers from a dissipative-soliton fiber laser," Optics Letters, vol. 34, no. 5, pp. 593-595, 2009.

[27] K. Tamura and M. Nakazawa, "Optimizing power extraction in stretched-pulse fiber ring lasers," Applied Physics Letters, vol. 67, no. 25, pp. 3691-3693, 1995.

[28] L. Q. Zhang, Z. Zhuo, Z. Y. Pan, Y. Z. Wang, J. W. Zhao, and J. X. Wang, "Investigation of pulse splitting behaviour in a dissipative soliton fibre laser," Laser Physics Letters, vol. 10, no. 10, Article ID 105104, 2013.

[29] M. J. Lederer, B. Luther-Davies, H. H. Tan, C. Jagadish, N. N. Akhmediev, and J. M. Soto-Crespo, "Multipulse operation of a Ti:sapphire laser mode locked by an ion-implanted semiconductor saturable-absorber mirror," Journal of the Optical Society of America B, vol. 16, no. 6, pp. 895-903, 1999. 

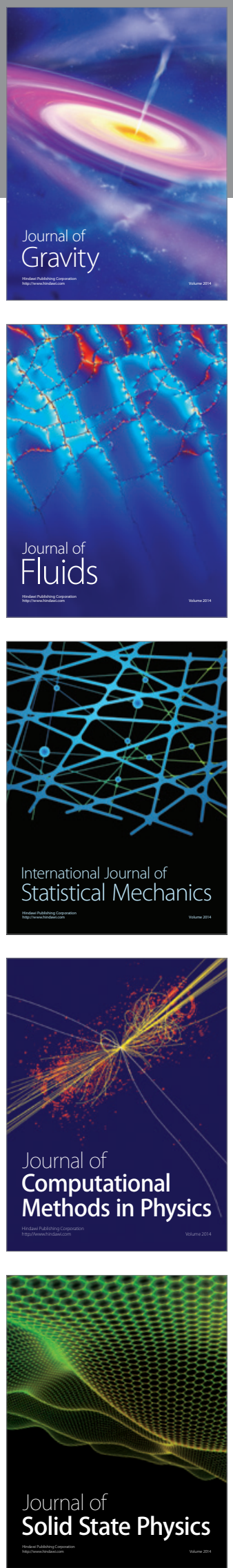

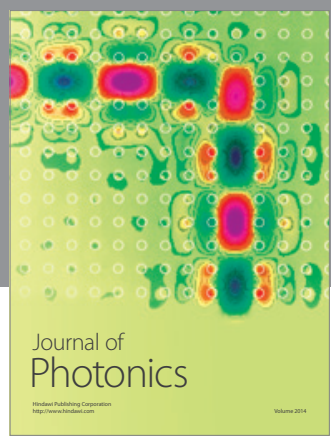

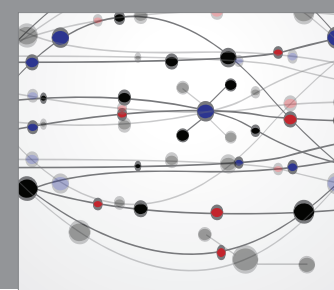

The Scientific World Journal

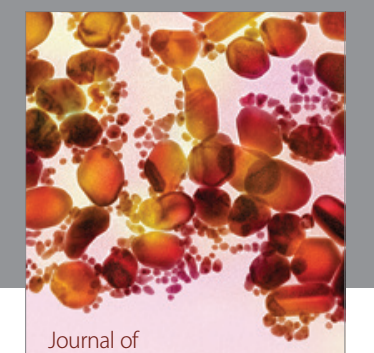

Soft Matter
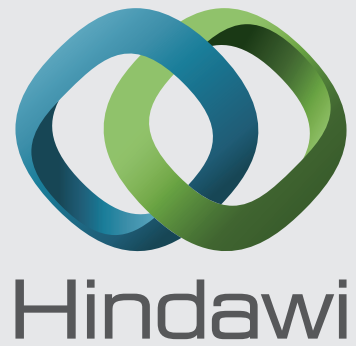

Submit your manuscripts at

http://www.hindawi.com
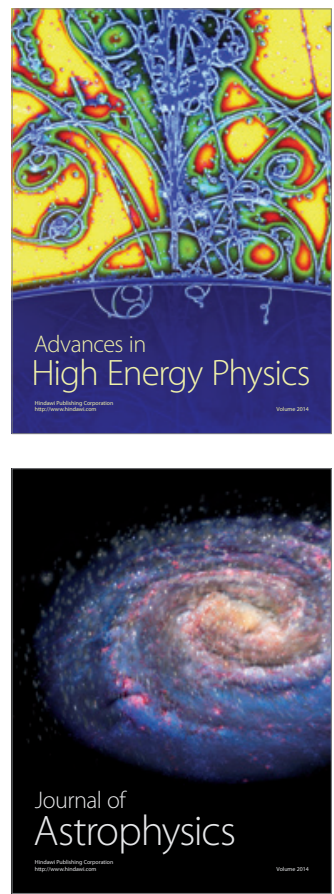
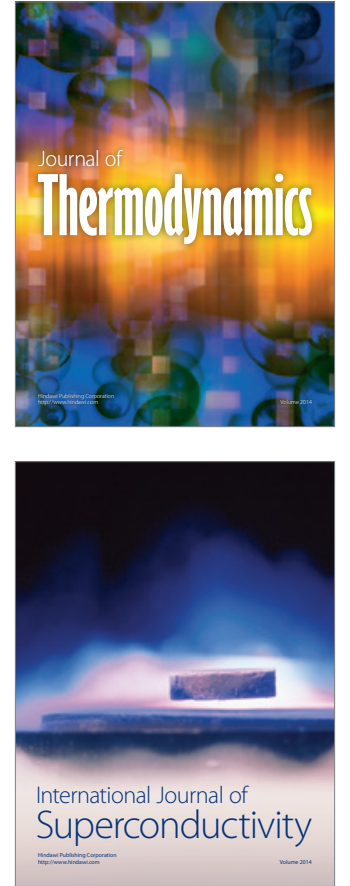
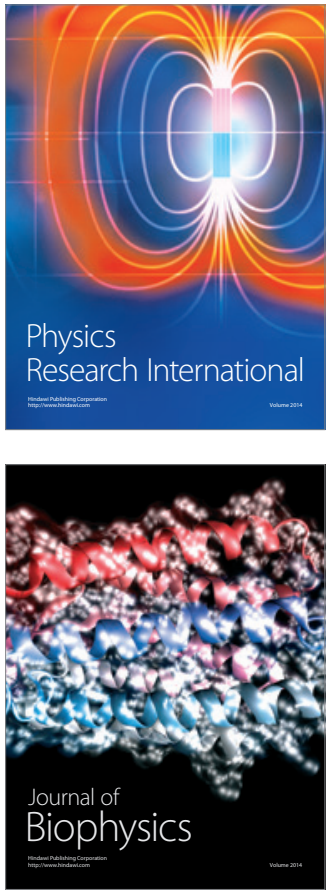
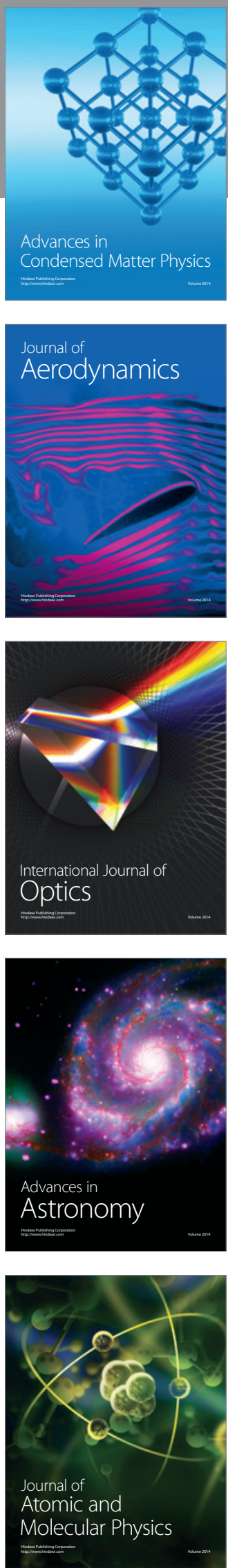